\title{
Interfaces da Enfermagem Dermatológica e Gerontológica na Prevenção de Lesões por Pressão: uma Reflexão
}

\author{
Dermatologic and Gerontological Nursing Interfaces \\ in Preventing Pressure Injuries: a Reflection
}

\author{
Interfaces de la Enfermería Dermatológica y Gerontológica \\ en la Prevención de las Lesiones por Presión: una Reflexión
}

Selma Petra Chaves Sá , Euzeli da Silva Brandão', Alessandra Conceição Leite Funchal Camacho', Beatriz Guitton Renaud Baptista de Oliveira ${ }^{1}$, Célia Pereira Caldas ${ }^{1}$

\begin{abstract}
RESUMO
Objetivo: Promover reflexão sobre a interface entre as especialidades de Enfermagem Dermatológica e Gerontológica visando à manutenção da integridade da pele do idoso, em especial na prevenção das lesões por pressão. Método: Trata-se de um artigo de reflexão abordando a importância da integração entre especialidades na tentativa de prevenir um problema que gera inúmeros prejuízos físicos, emocionais e sociais para o cliente, além do alto custo para as instituições. Resultados: As alterações cutâneas próprias do envelhecimento, associadas a outros fatores intrínsecos e extrínsecos, interferem na saúde da pele e podem predispor às lesões por pressão. Ao refletir especificamente sobre os fatores de risco que podem estar presentes no idoso, percebe-se a importância da avaliação interdisciplinar com vistas à integralidade do cuidado. Conclusão: Apesar das lesões por pressão não serem consideradas um agravo específico da pessoa idosa, torna-se imprescindível que os profissionais de saúde atentem para as especificidades do idoso. Nesse sentido, ressalta-se a contribuição da parceria entre as especialidades, nesse caso, Dermatologia e Gerontologia.
\end{abstract}

DESCRITORES: Estomaterapia. Enfermagem. Saúde de idoso. Dermatologia.

\begin{abstract}
Objective: To promote the reflection on the interface between the specialties of Dermatology and Gerontological Nursing for the maintenance of skin integrity in the elderly, especially in the prevention of pressure ulcers. Method: This is an article of reflection addressing the importance of the integration between specialties in an attempt to prevent a problem that causes immense physical, emotional and social harm to the client, besides high costs to institutions. Results: Aging skin changes, associated with other intrinsic and extrinsic factors, influence the health of the skin and may predispose to pressure ulcers. In reflecting specifically on the risk factors that may be present in the elderly, we realize the importance of interdisciplinary assessment with a view on comprehensive care. Conclusion: Despite pressure ulcers are not considered a specific grievance of the elderly, it is essential that health professionals take heed to the specificities of the elderly. In this sense, it should be highlighted the contribution of the partnership between the specialties, in this case, Dermatology and Geriatrics.
\end{abstract}

DESCRIPTORS: Stomatherapy. Nursing. Health of the elderly. Dermatology. 


\section{RESUMEN}

Objetivo: Promover la reflexión sobre la interfaz entre las especialidades de Enfermería Dermatología y Gerontológica para el mantenimiento de la integridad de la piel del añoso, en especial en la prevención de las lesiones por presión. Método: Se trata de un artículo de reflexión abordando la importancia de la integración entre las especialidades en el intento de prevenir un problema que genera innumerables daños físicos, emocionales y sociales para el cliente, además del alto costo para las instituciones. Resultados: Las alteraciones cutáneas propias del envejecimiento, asociadas a otros factores intrínsecos y extrínsecos, interfieren en la salud de la piel y pueden predisponer para lesiones por presión. Al reflexionar específicamente sobre los factores de riesgo que pueden estar presentes en el añoso, se nota la importancia de la evaluación interdisciplinaria con miras a la integralidad de la atención. Conclusión: A pesar de que las lesiones por presión no se consideran como una queja específica de las personas mayores, se vuelve imprescindible que los profesionales de la salud, estén atentos a las especificidades del añoso. En este sentido, se destaca la contribución de la asociación entre las especialidades en este caso, la Dermatología y la Geriatría.

DESCRIPTORES: Estomaterapia. Enfermería. Salud del añoso. Dermatología.

\section{INTRODUÇÃO}

Segundo projeções, desde 2010,11\% da população do país vêm sendo composta de pessoas acima de 60 anos $^{1}$, estando o Brasil à frente da Espanha em número de idosos².

O acelerado processo de envelhecimento populacional vem produzindo sérios desafios para o sistema de saúde, entre eles, o conhecimento por parte dos profissionais no que diz respeito às peculiaridades das pessoas que compõem esse grupo, impondo a necessidade premente de estabelecer estratégias para abordagem adequada às suas necessidades ${ }^{3}$.

Diante desse quadro, entre as diversas questões a serem discutidas, emerge a preocupação com a saúde da pele do idoso. Estudos revelam que mais de $90 \%$ dos idosos apresentam algum tipo de distúrbio na pele. Trata-se de um órgão complexo, que compreende $15 \%$ do peso corporal, no qual acontecem interações celulares e moleculares importantes, representando uma das demandas do envelhecimento com declínio progressivo na capacidade proliferativa e no tempo de vida das células 4 .

Com o envelhecimento, a capacidade desse órgão realizar as suas funções é reduzida. Nesse sentido, ressalta-se o significativo aumento do número de casos de problemas relacionados à pele, sejam eles inerentes especificamente ao próprio processo de envelhecimento da pele ou associados a outros agravos como diabetes, hipertensão, processos degenerativos, imobilidade e uso de medicamentos. Entre eles, citam-se o fotoenvelhecimento, o câncer de pele, as lesões por pressão e outros agravos, cuja incidência e prevalência aumentam com o passar da idade 5 .
Entre esses agravos, destaca-se neste artigo um desafio no cuidado de Enfermagem: a prevenção de lesões por pressão, tendo em vista que o desenvolvimento dessas lesões é considerado um problema mundial ${ }^{6}$ e sua incidência, um indicativo da qualidade dos cuidados.

A lesão por pressão é considerada um dano localizado na pele e/ou nos tecidos subjacentes, geralmente sobre uma proeminência óssea, relacionada ao uso de dispositivo médico ou outro, resultante da pressão intensa e/ou prolongada combinada ao cisalhamento, além da existência de outros fatores associados ${ }^{7}$. No Brasil, devido à precariedade de registros, a determinação da sua incidência e prevalência ainda é difícil. Um estudo retrospectivo realizado em prontuários de clientes hospitalizados em unidade de terapia intensiva de um hospital universitário de Recife, Pernambuco, revelou que de 56 clientes 24 desenvolveram lesões por pressão $(42,86 \%)^{8}$.

Apesar de o desenvolvimento dessas lesões não ser exclusivamente atribuído à questão da idade avançada, e sim a um conjunto de fatores associados, considera-se relevante destacar o risco aumentado nessa faixa etária, tendo em vista não somente a fragilidade da pele do idoso mas também a presença de comorbidades, uso de medicamentos, incontinência, entre outros fatores.

O desenvolvimento dessas lesões acarreta o aumento do tempo de hospitalização, de carga de trabalho para Enfermagem e dos custos, além de maior morbidade e mortalidade. Considerando a existência de fatores intrínsecos e extrínsecos, a avaliação do risco do cliente, em especial do idoso, torna-se imprescindível para prevenção dessas lesões. $\mathrm{Na}$ tentativa de facilitar essa avaliação, a literatura disponibiliza, há algumas décadas, escalas validadas na literatura como 
Norton, Waterlow e Braden. Apesar da disponibilidade desses recursos, a sua aplicação na prática ainda é um desafio 6 .

Nesse contexto, considera-se importante lembrar as responsabilidades e os deveres do enfermeiro conforme cita o art. 12 da Resolução no 311/2007 do Conselho Federal de Enfermagem (COFEN) de assegurar à pessoa, à família e à coletividade assistência de enfermagem livre de danos decorrentes de imperícia, negligência ou imprudência ${ }^{9}$.

No que diz respeito especificamente ao idoso, lembra-se a Portaria no 1.395/99 da Política de Saúde do Idoso, que preconiza a manutenção da sua capacidade funcional, ou seja, a manutenção das habilidades físicas e mentais necessárias para uma vida independente e autônoma ${ }^{10}$. Assim, compreende-se que o desenvolvimento de lesão por pressão representa para o idoso a perda de sua capacidade funcional, dificultando ou até mesmo impedindo a realização das atividades de vida diária, acarretando, assim, maior dependência.

Considerando que as alterações que ocorrem no processo de envelhecimento devem ser acompanhadas por diferentes membros da equipe multidisciplinar, incluindo as diversas questões que envolvem a manutenção e a recuperação da integridade da pele, mencionam-se as especialidades de Enfermagem em Dermatologia e Gerontologia, ambas reconhecidas na lista de especialidades no âmbito do Sistema COFEN/Conselhos Regionais de Enfermagem, Resolução no 389, de 18 de outubro de $2011^{11}$. Destaca-se ainda a existência da Associação Brasileira de Enfermagem em Dermatologia (SOBENDE) e da Associação Brasileira de Estomaterapia (SOBEST) ${ }^{12}$, entidades civis sem fins lucrativos e de caráter científico cultural, que têm como um de seus objetivos agregar enfermeiros na área da assistência, ensino e pesquisa para promover o desenvolvimento cientifico, viando à melhoria da qualidade do atendimento.

No caso do idoso, busca-se entender melhor o processo de envelhecimento, a fim de planejar medidas mais adequadas para promoção da saúde, prevenção de agravos, principalmente em relação à manutenção e/ou recuperação da integridade da pele das pessoas nessa fase específica da vida ${ }^{5}$.

Nesse contexto, destaca-se a Enfermagem Gerontológica, que tem a sua ênfase na maximização do nível de independência do indivíduo para o desenvolvimento de suas atividades de vida diária, em prevenir as doenças e promover, manter e restaurar a saúde, e em preservar a dignidade, o conforto e o bem-estar do idoso ${ }^{13}$.

Diante dessas considerações, este artigo tem como objetivo promover a reflexão sobre a interface entre as especialidades de Enfermagem em Dermatologia e em Gerontologia, visando à manutenção da integridade da pele do idoso, em especial na prevenção das lesões por pressão.

\section{MÉTODO}

Trata-se de um artigo de reflexão abordando a importância da integração entre as especialidades Dermatologia e Gerontologia, na tentativa de desenvolver ações conjuntas para prevenir um problema que gera inúmeros prejuízos físicos, emocionais e sociais para o cliente, além do alto custo para as instituições.

\section{INTERFACES ENTRE ESPECIALIDADES: ENFERMAGEM DERMATOLÓGICA E GERONTOLÓGICA NA PREVENÇÃO DE LESÕES POR PRESSÃO NO IDOSO}

Embora ao longo dos anos a ocorrência das lesões por pressão tenha sido equivocadamente atribuída exclusivamente aos fatores extrínsecos, ou seja, relacionados diretamente aos cuidados de Enfermagem, atualmente reconhece-se a influência de fatores intrínsecos, que são relacionados ao quadro clínico do cliente e que necessitam ser avaliados pelo enfermeiro para implementação de medidas corretivas e preventivas ${ }^{14}$.

Assim, durante a avaliação do idoso hospitalizado ou não, o enfermeiro deve considerar na entrevista e no exame físico as condições biopsicossocial, espiritual e econômica, com ênfase no estado geral e cognitivo, as condições posturais e de locomoção, os sinais vitais e nutricionais, assim como a presença de enfermidades e o uso de medicamentos que podem interferir na sua mobilidade, sensibilidade e/ou na circulação periférica. Nesse contexto, considera-se importante citar os recursos disponíveis para a avaliação das condições cognitivas, capacidade funcional, atividades de vida diária e instrumentais, como a escala do Mini Exame do Estado Mental, Katz e Lawton ${ }^{15}$.

No que diz respeito especificamente ao exame da pele, destacam-se a inspeção, caracterizada pela visualização geral 
da superfície da pele, e a palpação, que auxilia na detecção de alterações. $\mathrm{O}$ exame minucioso e atento é indispensável para identificar alterações na cor, textura, temperatura, turgor, presença de edema, de umidade e de lesões ${ }^{16}$. Importa ainda mencionar a importância de considerar fatores como etnia e atividade laboral que o idoso exerceu e/ou que ainda exerce.

O envelhecimento provoca alterações fisiológicas e estruturais em praticamente todos os sistemas do corpo, em grande parte afetado por fatores genéticos, dieta, condições sociais e doenças. Sobre as alterações próprias do envelhecimento, alerta-se que o idoso apresenta a pele mais seca devido à redução da quantidade e da atividade das glândulas sebáceas e exócrinas. A redução do sebo aumenta o ressecamento e reduz a proteção da pele, já que este apresenta ação fungicida. A redução do tecido subcutâneo, responsável pela sustentação, auxilia no enrugamento da pele, além de facilitar a ocorrência de lesões ${ }^{13}$.

A redução das células de Langerhans, responsáveis pela fagocitose de antígenos, torna o idoso ainda mais susceptível aos microrganismos. Devido à perda de 10 a 20\% de melanócitos em cada década de vida, os idosos têm menor proteção contra os raios ultravioleta (UV), que induzem mutações celulares ${ }^{17}$.

Os vasos sanguíneos apresentam maior fragilidade. A redução da vascularização cutânea diminui a temperatura da pele e causa palidez. Alterações nas terminações nervosas diminuem a sensibilidade da pele, facilitando a ocorrência de traumatismos ${ }^{17,18}$

O déficit sensorial diminui a capacidade de responder aos estímulos álgicos. As doenças agudas, crônicas, graves ou terminais diminuem ainda mais o fornecimento de sangue para a periferia e, consequentemente, o aporte de nutrientes e oxigênio para os tecidos ${ }^{19}$.

O uso de medicamentos, como drogas vasoativas, esteroides, citotóxicas e imunossupressoras, reduz a resistência dos tecidos. A história prévia de lesões por pressão reduz a resistência do tecido presente na região previamente acometida ${ }^{19}$.

Quando presente, a deficiência nutricional pode causar anemia, reduzir a oferta de nutrientes e oxigênio para os tecidos e a elasticidade da pele. Nesse sentido, alerta-se que os idosos emagrecidos possuem menos proteção contra pressão. Por outro lado, a obesidade dificulta o deslocamento durante a mobilização ${ }^{19}$.

A diminuição do nível de consciência, as fraturas e o desenvolvimento de doenças leva à redução da mobilidade ou imobilidade do idoso. A redução da mobilidade pode ser agravada com o uso de ansiolíticos, analgésicos e sedativos, realização de procedimentos cirúrgicos, entre outros ${ }^{19}$.

Ressalta-se, ainda, que as incontinências podem estar presentes, sendo a urinária mais comum entre as mulheres idosas ${ }^{13}$.

Sobre os fatores extrínsecos, destacam-se a pressão, o cisalhamento, a maceração e a fricção. Assim, ressalta-se que as alterações cutâneas próprias do envelhecimento, associadas a outros fatores intrínsecos e extrínsecos que podem estar presentes, interferem na saúde da pele e podem predispor às lesões por pressão. Ao refletir especificamente sobre os fatores de risco apresentados pelos idosos, percebe-se a necessidade de valorizar a identificação de tais fatores e a implementação de ações corretivas e preventivas personalizadas.

No que diz respeito à avaliação dos indivíduos em risco, primeiro aspecto na perspectiva da prevenção, considera-se importante mencionar que o primeiro modelo de indicador de risco foi desenvolvido em 1975, o Norton Score. Norton estudou clientes geriátricos e estabeleceu cinco fatores: estados físico e mental, atividade, mobilidade e incontinência. Cada fator foi dividido em quatro níveis e foi atribuída uma nota. O total das cinco notas permite identificar o grau de risco. Quanto maior a nota, menor o risco ${ }^{19,20}$.

Posteriormente, outros indicadores de risco foram sugeridos e validados, entre eles a Escala de Braden, que é composta por seis domínios: percepção sensorial, umidade, atividade, mobilidade, nutrição, fricção e cisalhamento. Cinco domínios são pontuados de 1 a 4, exceto fricção e cisalhamento, com pontuação de 1 a 3. Cada domínio é acompanhado por uma breve descrição dos critérios que deverão ser considerados pelos avaliadores, conforme suas observações clínicas. Ao somar os escores dos 6 domínios, chega-se a um escore de risco que varia de 6 a 23, e a pontuação mais baixa indica maior risco ${ }^{19,20}$.

Apesar de o Norton Score estar mais voltado para avaliação do risco do idoso, ressalta-se que nenhum instrumento de avaliação é considerado o melhor ou o mais apropriado. Recomenda-se a utilização de instrumento validado e adequado à realidade de cada contexto assistencial ${ }^{20}$.

Objetivando caracterizar formas de atuar preventivamente no desenvolvimento das lesões de pressão, a National Pressure Ulcer Advisory Panel, a European Pressure Ulcer Advisory Panel e a Pan Pacific Pressure Injury Alliance elaboraram um guia para prevenção e tratamento de lesões por pressão. Especificamente sobre os clientes idosos, as recomendações existentes nesse documento destinam-se a complementar, e não a substituir, as recomendações 
preventivas gerais. Nesse sentido, destacam aspectos relacionados à avaliação e ao planejamento dos cuidados, ressaltando a avaliação do estado cognitivo, a diferenciação com outros tipos de lesões comuns nos idosos, a importância do envolvimento da família e a educação ao indivíduo/família. Sobre os cuidados com a pele envelhecida e vulnerável, lembram a proteção da pele vulnerável à pressão e ao cisalhamento, o uso de produtos e a implementação de plano de cuidados relacionado à incontinência. Quanto ao reposicionamento, chamam a atenção sobre o reposicionamento regular do idoso dependente, a redistribuição da pressão, a atenção na posição e na técnica de movimentação manual, além do reposicionamento frequente da cabeça dos idosos que estejam sedados, ventilados ou imobilizados ${ }^{21}$.

\section{CONCLUSÃO}

Diante do envelhecimento populacional crescente e de toda a reflexão apresentada, pode-se considerar que a prevenção de lesões por pressão no idoso é uma temática de vasta abrangência e, portanto, de responsabilidade de todos os profissionais de saúde, além dos cuidadores formais e não formais.

Apesar das lesões por pressão não serem consideradas um agravo específico da pessoa idosa, torna-se imprescindível que os profissionais de saúde atentem para as especificidades do idoso. Assim, alerta-se para a relevância do compartilhar conhecimentos visando à prevenção dessas lesões.

Nesse contexto, ressaltam-se as contribuições dos especialistas em Dermatologia e Gerontologia que, diante do seu olhar especializado, podem ser agentes facilitadores/ multiplicadores no sentido de: avaliar o idoso de forma conjunta visando à interdisciplinaridade e integralidade do cuidado; criar estratégias para incrementar a aplicação das escalas preditivas e respectivas medidas preventivas; realizar ações educativas com os profissionais de saúde, cuidadores de idosos formais ou não formais; responder pareceres; implementar ações preventivas; promover a interação idoso-profissional-cuidador, indispensável para auxiliar a aceitação e realização das ações recomendadas; contribuir para a realização de pesquisas em médio e longo prazo, facilitando a caracterização dos fatores de riscos específicos apresentados por essa clientela e a efetividade das ações implementadas.

\section{REFERÊNCIAS}

1. Instituto Brasileiro de Geografia e Estatística (IBGE). Perfil dos idosos responsáveis pelos domicílios [Internet]. Brasília: IBGE, 2002 [cited 2016 Ago 02]. Available from: http://www.ibge. gov.br/home/presidencia/noticias/25072002pidoso.shtm

2. Ferreira JDL, Aguiar ESS, Soares MJGO, Silva MA, Oliveira SHS. Risco e prevalência de úlcera por pressão em usuários da rede básica de saúde. Rev Enferm UFPE on line. 2012;6(9):2045-51.

3. Telles $J \mathrm{~L}$, Borges APA. Interdisciplinaridade e trabalho em equipe na abordagem da pessoa idosa nos serviços de atenção básica em saúde. In: Malagutti W, Bergo AMA. Abordagem interdisciplinar do idoso. Rio de Janeiro: Rubio, 2010. p. 1-7.

4. Roach SS. Introdução à enfermagem gerontológica. Rio de Janeiro: Guanabara Koogan; 2009.

5. Brandão ES, Santana RF, Mandelbaum MH. Cuidados gerais ao idoso com vitiligo. In: Malagutti W, Bergo AMA. Abordagem interdisciplinar do idoso. Rio de Janeiro: Rubio, 2010. p. 235-54.

5. Mandelbaum SH, Santis EPD, Mandelbaum MHS Cicatrização: conceitos atuais e recursos auxiliares - Parte I. An Bras Dermatol. 2003;78(4):393-410.

6. Brandão ES, Mandelbaum MHS, Santos I. Um desafio no cuidado em enfermagem: prevenir úlceras por pressão no cliente. Rev Pesq Cuid Fundam (Online). 2012; 6(8):1965-70.

7. National Pressure Ulcer Advisory Panel [Internet]. National Pressure Ulcer Advisory Panel (NPUAP) announces a change in terminology from pressure ulcer to pressure injury and updates the stages of pressure injury. [Internet]. 2016 [cited 2016 Ago 02]. Avaiable from: http://www.npuap.org/ national-pressure-ulcer-advisory-panel-npuap-announcesa-change-in-terminology-from-pressure-ulcer-to-pressureinjury-and-updates-the-stages-of-pressure-injury/

8. Santos JGN, Carvalho PO, Vieira JCM. Profile of patients with pressure ulcers in the intensive care unit. J Nurs UFPE on line. 2012;6(2):378-85.

9. Conselho Federal de Enfermagem. Resolução n 311, de 9 de fevereiro de 2007. Código de Ética dos Profissionais de Enfermagem. Brasília: COFEN; 2007.

10. Brasil. Ministério da Saúde. Portaria no 1.395, de 10 de dezembro de 1999. Política Nacional de Saúde do Idoso. Brasília: Ministério da Saúde; 1999.

11. Conselho Federal de Enfermagem. Resolução COFEN nº 389, de 18 de outubro de 2011. Atualiza, no âmbito do Sistema COFEN/Conselhos Regionais de Enfermagem, os procedimentos para registro de título de pós-graduação lato e stricto sensu concedido a enfermeiros e lista as especialidades. Brasília: COFEN; 2011

12. Ferreira AM, Bogamil DDD, Tormenta PC. O enfermeiro e o tratamento de feridas: em busca da autonomia do cuidado. Arq Cienc Saúde. 2008;15(3):105-9.

13. Leal LS. O papel do enfermeiro e a assistência à pele integra do idoso. In: Malagutti W, Bergo AMA. Abordagem interdisciplinar do idoso. Rio de Janeiro: Rubio; 2010. p. 223-33. 
14. Brandão ES, Santos JA, Santos I. Úlceras por compressão: importância da avaliação do cliente. In: Silva RCL, Figueiredo NMA, Meireles IB. Feridas: fundamentos e atualizações em enfermagem. 3 ed. São Paulo: Yendys Editora; 2011. p. 405-12.

15. Lino VTS, Pereira SRM, Camacho LAB, Ribeiro Filho ST, Buksman S. Adaptação transcultural da Escala de Independência em Atividades da Vida Diária (Escala de Katz). Cad. Saúde Pública. 2008;24(1):103-12.

16. Rotta O. Propedêutica clinica. In: Rotta O. Dermatologia: clínica, cirúrgica e cosmiátrica. São Paulo: Manole; 2008. p.13-25.

17. Roach SS. Introdução à enfermagem gerontológica. Rio de Janeiro: Guanabara Koogan; 2009.
18. Eliopoulos C. Enfermagem gerontológica. Porto Alegre: Artmed; 2005.

19. Santos JA, Brandão ES. Clientes com úlceras por pressão: prevenção, avaliação e tratamento. In: Brandão ES, Santos I. Enfermagem em dermatologia: cuidados técnico, dialógico e solidário. Rio de Janeiro: Cultura Médica; 2006. p. 257-79.

20. Blanes L, Yosbitome AY, Ferreira LM. Úlcera por pressão: utilizando instrumentos de avaliação de risco como estratégia para prevenção. Estima; 2003;1(3):37-45.

21. National Pressure Ulcer Advisory Panel, European Pressure Ulcer Advisory Panel and Pan Pacific Pressure Injury Alliance. Prevention and treatment of pressure ulcers: quick reference guide. Emily Haesler (Ed.). Osborne Park: Cambridge Media; 2014. 